\title{
BEAM DYNAMICS FOR THE PRELIMINARY PHASE OF THE NEW CLIC TEST FACILITY (CTF3)
}

\author{
R. Corsini, A. Ferrari, L. Rinolfi, T. Risselada, P. Royer, F. Tecker \\ CERN, Geneva, Switzerland
}

\begin{abstract}
In the framework of the CLIC (Compact Linear Collider) $\mathrm{RF}$ power source studies, the scheme of electron pulse compression and bunch frequency multiplication, using injection by RF deflectors into an isochronous ring, will be tested, at low charge, during the preliminary phase of the new CLIC Test Facility (CTF3) at CERN. In this paper, we describe the beam dynamics studies made in order to assess the feasibility of the bunch combination experiment, as well as the related beam measurements performed on the LEP Pre-Injector complex (LPI) before its transformation into CTF3.
\end{abstract}

\section{INTRODUCTION}

The new CLIC Test Facility (CTF3) $[1,2]$ is being built at CERN in the LPI area. The aim of this facility is to demonstrate the technical feasibility of the CLIC RF power source by generating a $30 \mathrm{GHz}$ RF pulse with the CLIC nominal peak power and pulse length [3]. The scheme uses electron pulse compression and bunch frequency multiplication based on the combination of electron bunch trains in isochronous rings using RF deflectors for injection. The construction of CTF3 is scheduled in stages over five years. As a preliminary phase, to begin in September 2001, it is planned to test the bunch combination scheme at low charge [4], making use of the existing LPI components.

In the following, the beam dynamics studies for this preliminary phase of CTF3 [5] are described and the related beam measurements performed on the LPI installation are reported.

\section{BEAM DYNAMICS STUDIES}

The general layout of the CTF3 preliminary phase is shown in Figure 1. A pulse train of up to five pulses is produced at the source in the front-end, is then accelerated in a linac and injected into an isochronous ring. Each pulse is composed of 20 electron bunches, spaced by $10 \mathrm{~cm}$ (corresponding to the $3 \mathrm{GHz}$ acceleration frequency). The distance between the pulses corresponds to the ring circumference, so that the pulses can be combined turn after turn at injection, by interleaving their bunches using RF deflectors. A single pulse with up to five times the initial pulse charge and up to five times the bunch repetition frequency will thus be obtained after combination.

\subsection{The Front-End}

Since the former LEP Injector Linac (LIL) thermionic gun is not adapted to the CTF3 requirements, a collaboration with the "Laboratoire de l'Accélérateur Linéaire" (LAL, Orsay) has been established to build a new thermionic gun similar to the one used in the CLIO machine [6]. This triode gun produces a train of up to seven pulses with a repetition rate of $50 \mathrm{~Hz}$. The nominal length of each pulse is $6.6 \mathrm{~ns}$ FWHM, and the pulses are spaced by $420 \mathrm{~ns}$, corresponding to the ring circumference. The gun operates with a nominal peak current of $1 \mathrm{~A}$. At the exit of the gun, the LIL bunching system, composed of a single-cell pre-buncher and a standing wave buncher, is powered independently by a $30 \mathrm{MW}$ klystron and produces a $3 \mathrm{GHz}$ bunched beam at $4 \mathrm{MeV}$. Each pulse then consists of 20 bunches, each bunch having a charge of $0.1 \mathrm{nC}$ and a length of about $10 \mathrm{ps}$ FWHM. The transverse matching section between the front-end and the linac is made of one solenoid and one quadrupole triplet, with independent power supplies.

\subsection{The Linac}

Some modifications of the former linac are mandatory: the first part of LIL is dismantled, eight of the sixteen accelerating structures are removed, the existing bunching system with the new gun is moved downstream, and a rearrangement of the remaining components is required. The pulse train is then accelerated to the reference energy of $350 \mathrm{MeV}$, using eight travelling wave accelerating structures which are powered in groups of four by two $45 \mathrm{MW}$ klystrons. The whole electron pulse train is accelerated using a single RF pulse. The beam parameters have been chosen in order to minimize the energy spread generated by beam-loading in the LIL structures, while still keeping a charge per bunch high enough to give a good resolution for the measurements of the beam time structure with a streak camera. Because of beam-loading, the total energy spread is about $6 \mathrm{MeV}$, which is within the ring full acceptance $( \pm 1 \%)$. It can nevertheless be reduced by a factor of two by delayed RF filling of the structures, or by dumping the first two pulses out of seven in the train. Indeed, the frequency multiplication test requires at most five pulses. The transverse matching to the injection line is done at the end of the linac with five independent quadrupoles. This matching section is instrumented to provide a full characterization of the beam, with time-resolved measurements of the transverse parameters and of the beam energy spectrum using Transition-Cherenkov monitors. 


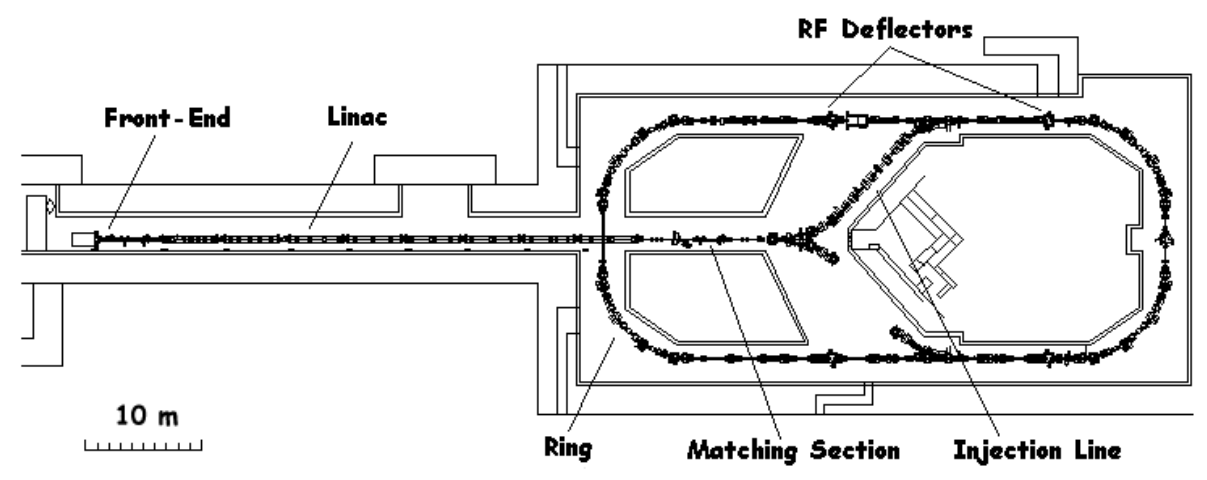

Figure 1: Layout of the CTF3 preliminary phase complex.

\subsection{The Injection Line}

The transfer line from the linac to the ring must be isochronous and achromatic in both planes (in addition to the main horizontal bending magnets, the line contains two vertical dipoles to match the heights of the linac and of the ring). Isochronicity is mandatory in order to preserve the bunch length from the linac to the ring. This is essential for the combination process with the RF deflectors, for which short bunches $(\leq 15$ ps FWHM $)$ are needed. To achieve the isochronicity condition and the transverse matching to the new ring lattice, two new quadrupoles are added and the positions and gradients of the existing quadrupoles are modified. The overall geometry of the line is preserved but all these quadrupoles need independent power supplies. This configuration allows a quasi-isochronous operation with $\left|R_{56}\right| \leq 10^{-2} \mathrm{~m}$ where the $R_{56}$ factor is the standard linear dependence of the path length with the energy spread. The second order in isochronicity is small and no sextupole corrections are needed in the transfer line.

\subsection{The Ring}

The lattice of the existing Electron-Positron Accumulator (EPA) ring is modified to make the ring isochronous (i.e with a zero momentum compaction factor), thus preserving the bunch length and spacing during the combination process (up to five turns). In the new isochronous lattice, the dispersion vanishes in the long straight sections. This new optics requires the displacement of four quadrupoles and the decoupling of three of the six existing quadrupole families. Although the symmetry with respect to the centre of each of the four arcs is abandoned, the symmetry of the ring with respect to the centre of each of the four straight sections between the arcs is preserved. By choosing appropriate integer tune values, the required gradients are kept at a moderate level, and the new isochronous optics can be achieved with the present magnets. In order to better match the arc optics to the straight section optics, one quadrupole in each arc is displaced by $1.85 \mathrm{~m}$ towards the long straight section. The natural chromaticities of the new isochronous lattice are comparable with those of the former EPA lattice. Equally, the off-momentum path lengths can be controlled using the chromaticity sextupoles (organized in three families) to within $0.5 \mathrm{~mm}$ per turn across the momentum range of the bunches, while keeping the chromaticities small. Tracking over 1000 turns shows horizontal and vertical dynamic apertures around $15 \sigma$ across the momentum range of the incoming beam. Combination factors of three, four and five will be tested in the preliminary phase of CTF3. With a given ring layout, the different combination factors can be explored by changing the frequency of both the linac and the RF deflectors by $\pm 150 \mathrm{kHz}$. In order to follow the change of the RF frequency the accelerating structures and the deflectors are tuned in operation by varying their temperature by $\pm 3^{\circ} \mathrm{C}$ [7]. The ring circumference is fixed to an average value, corresponding to a combination factor four. This requires a reduction of $17 \mathrm{~mm}$ of the ring circumference (whose nominal value is $40 \pi \mathrm{m}$, as recently confirmed [7]). Apart from the standard ring instrumentation, the main diagnostic tool is the streak camera which is used to observe the beam structure and to provide the demonstration of the bunch combination.

\subsection{The Injection using RF Deflectors}

In order to achieve bunch frequency multiplication, the bunch trains are injected into the isochronous ring using two RF deflectors. The deflectors are located in the ring with a betatron phase advance of $\pi$ between them, so that they produce a time-dependent closed bump of the reference orbit, allowing the interleaving of three to five bunch trains. The RF deflectors are travelling wave, iris-loaded structures for which the fundamental mode is a deflecting mode, with a phase advance of $\pi / 2$ per cell and a negative group velocity. They are powered by one of the existing $30 \mathrm{MW}$ klystrons, and a power of about $7 \mathrm{MW}$ per cavity is needed to obtain the nominal deflecting angle of $4.5 \mathrm{mrad}$ at $350 \mathrm{MeV}$. Figure 2 shows the variation of the transverse kick for the injected and the circulating bunches. The corresponding beam envelope simulations are shown in Figure 3. The fast injection kickers are kept in the ring to allow conventional single-turn injection, for checking the ring optics prior to the installation of the RF deflectors. Another kicker remains to extract the circulating beam to a dump located in the former positron injection line. 


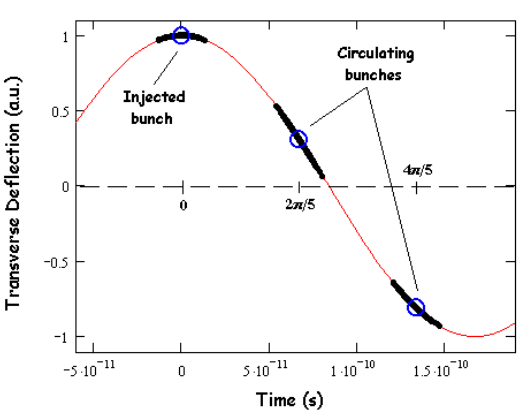

Figure 2: Kick amplitudes for the injected and the first two circulating bunches for a combination factor of five. The extension corresponds to $\pm 2 \sigma$ of a Gaussian distribution with a length of $10 \mathrm{ps}$ FWHM.

\section{RELATED BEAM MEASUREMENTS}

Recent isochronicity tests were performed in the EPA ring to demonstrate the capability to control the momentum compaction factor to the level required for the preliminary phase of CTF3. The tests were made with a charge of $2.5 \mathrm{nC}$ per pulse and with an energy close to the nominal one (308 MeV instead of $350 \mathrm{MeV}$ ) [7]. The isochronous lattice was obtained by changing the strength of the existing quadrupole families. However, the dispersion did not vanish in the long straight sections and the transfer line was badly matched to the ring, causing beam losses in the first few turns. Nevertheless, streak camera images showed the bunch structure unchanged up to the $40^{\text {th }}$ turn in the ring, compared to only a few turns with the nominal optics. After one turn in the ring, the measured bunch length is $33 \mathrm{ps}$ FWHM, due to bunch lengthening in the non-isochronous transfer line, compared to 66 ps FWHM with the nominal optics in the ring. These values are fully consistent with a bunch length of 7 ps FWHM at the end of the linac, as previously measured [8].

In order to validate a new model of the existing linac, which will be used during the commissioning of the preliminary phase of CTF3, quadrupole scans were performed with different acceleration conditions to characterize the Twiss parameters and the energy gains along the linac [7]. Trajectory analysis allowed a better understanding of the energy gains in the structures. The simulations are now in very good agreement with the measurements for the horizontal plane, but a small discrepancy remains to be understood in the vertical plane, though not critical.

The exploration of the whole range of combination factors relies on the fact that the LIL accelerating structures can be tuned in operation by varying their temperature, in order to follow the change of the RF frequency. Recent measurements [7] have shown that the dependence of the resonant frequency of the cavities on the temperature is as predicted by theory and that no measurable effects are observed on the beam when following the frequency change with the temperature.

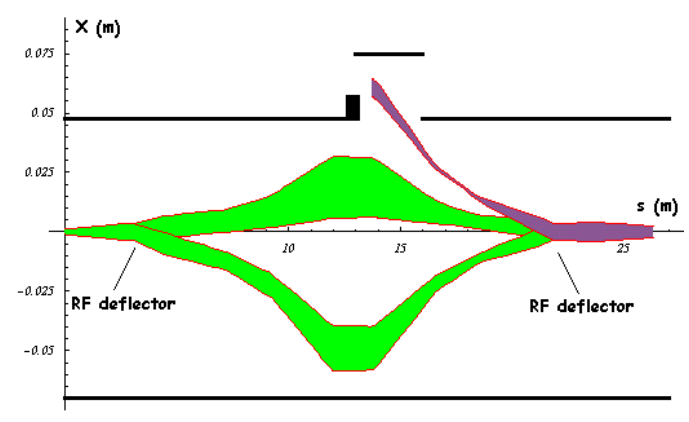

Figure 3: Beam envelopes of the bunches of Figure 2. From top to bottom: injected bunch, circulating bunches with phase $\frac{2 \pi}{5}$ and $\frac{4 \pi}{5}$. The lines are the limits of the vacuum chamber and the rectangle stands for the injection septum.

\section{CONCLUSION}

The beam dynamics studies described in this paper take into account analytical and numerical calculations, as well as results of a series of beam measurements made on the LPI installation. This analysis allowed us to fix the layout and the optics of the new CLIC Test Facility CTF3 for its preliminary phase. In addition, the required hardware modifications of the former LPI complex were identified. These modifications have begun, and the commissioning is expected to begin in September 2001, prior to the bunch combination tests.

\section{REFERENCES}

[1] G. Guignard (Ed.), "A $3 \mathrm{TeV} e^{+} / e^{-}$Linear Collider Based on CLIC Technology”, CERN 2000-008 (2000).

[2] CLIC Study Team, "Proposals for Future CLIC Studies and a New CLIC Test Facility (CTF3)", CERN/PS 99-047 (LP) and CLIC Note 402 (1999).

[3] R. Corsini (Ed.), "The CLIC RF power source: a novel scheme of two beam acceleration for electron-positron linear colliders", CERN 99-06 (1999).

[4] R. Corsini, A. Ferrari, J.P. Potier, L. Rinolfi, T. Risselada, P. Royer, "A Low Charge Demonstration of Electron Pulse Compression for the CLIC RF Power Source", Proc. 20th International Linear Accelerator Conference, Monterey, USA, 2000.

[5] R. Corsini, A. Ferrari, L. Rinolfi, T. Risselada, P. Royer, F. Tecker, "Beam Dynamics for the CTF3 Preliminary Phase", CLIC Note 470 (2001).

[6] J.C. Bourdon, R. Belbeoch, M. Bernard, P. Brunet, B. Leblond, M. Omeich, E. Plouviez, J. Rodier, "Commissioning the CLIO injection system", Nucl. Instr. Meth. in Physics Research A 304, 322-328 (1991).

[7] R. Corsini, B. Dupuy, A. Ferrari, L. Rinolfi, T. Risselada, P. Royer, F. Tecker, "LIL Lattice Parameters, LIL Energy Gains, LIL Temperature vs RF Frequency, EPA Circumference and EPA Isochronicity Measurements", CTF3 note 2000-18 (MD).

[8] R. Corsini, A. Ferrari, L. Rinolfi, T. Risselada, P. Royer, F. Tecker, "LIL Bunch Length and Lattice Parameters Measurements in March 2000", CTF3 note 2000-09 (MD). 